\title{
Captures
}

Figures, théories et pratiques de l'imaginaire

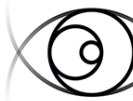

C A P T U R E S

\section{Littérature suspecte}

Ambiguiités, tromperies, détournements

\section{Cassie Bérard and Jean-Philippe Lamarche}

Volume 3, Number 2, November 2018

Littérature suspecte

URI: https://id.erudit.org/iderudit/1055822ar

DOI: https://doi.org/10.7202/1055822ar

See table of contents

Publisher(s)

Figura, Centre de recherche sur le texte et l'imaginaire

ISSN

2371-1930 (digital)

Explore this journal

Cite this document

Bérard, C. \& Lamarche, J.-P. (2018). Littérature suspecte : ambiguïtés, tromperies, détournements. Captures, 3(2). https://doi.org/10.7202/1055822ar
Article abstract

Literature hates accomplices. To think of the reading experience in these terms allows us to glimpse at some of the distance effects generated by a part of the narrative literature of the 20th and 21st centuries, which is held in suspicion. This emancipation bias - where the text becomes autonomous and the narrative becomes more complex to the point of concealing, stifling or even destroying its own mechanics - also pushes us to challenge certain aspects of fiction: is there, in the end, in "playful feinty", an honest sharing between the author and the reader?
Tous droits réservés (C Cassie Bérard, Jean-Philippe Lamarche, 2018

\section{(c) (i) $\Theta$}

This document is protected by copyright law. Use of the services of Érudit (including reproduction) is subject to its terms and conditions, which can be viewed online.

https://apropos.erudit.org/en/users/policy-on-use/ 


\section{Littérature suspecte}

\section{Ambiguïtés, tromperies, détournements}

\section{Cassie Bérard \\ Jean-Philippe Lamarche}

Résumé :

La littérature a horreur des complices. Penser l'expérience de lecture en ces termes permet d'entrevoir certains effets de mise à distance que génère un pan de la littérature narrative des $X X^{e}$ et $X X l^{e}$ siècles, tenu en suspicion. Ce parti pris d'émancipation — où le texte s'autonomise et le récit se complexifie jusqu'à dissimuler, étouffer, voire anéantir sa propre mécanique - pousse aussi à contester certains ressorts de la fiction : y a-t-il, en fin de compte, dans la « feintise ludique », un partage honnête entre l'auteur et le lecteur?

Literature hates accomplices. To think of the reading experience in these terms allows us to glimpse at some of the distance effects generated by a part of the narrative literature of the $20^{\text {th }}$ and $21^{\text {st }}$ centuries, which is held in suspicion. This emancipation bias - where the text becomes autonomous and the narrative becomes more complex to the point of concealing, stifling or even destroying its own mechanics - also pushes us to challenge certain aspects of fiction: is there, in the end, in "playful feinty", an honest sharing between the author and the reader?

La littérature a horreur des complices. Penser l'expérience de lecture en ces termes permet d'entrevoir certains effets de mise à distance que génère un pan de la littérature narrative des $X X^{e}$ et $X X l^{e}$ siècles tenu en suspicion. Ce parti pris d'émancipation grâce auquel le texte s'autonomise et le récit se complexifie jusqu'à dissimuler, étouffer, voire anéantir sa propre mécanique, pousse aussi à contester certains ressorts de la fiction : y a-t-il, en fin de compte, dans la « feintise ludique » (Schaeffer, 1999), un partage honnête entre l'auteur et le lecteur? Nathalie Sarraute annonçait, dès 1956 dans L'ère du soupçon, de possibles débordements : en plus de se méfier des personnages, le lecteur et l'auteur allaient dorénavant se méfier l'un de l'autre. C'est à ce phénomène de méfiance exacerbée, largement répandu aujourd'hui dans la création littéraire et dans la théorie critique, que le dossier sur la « Littérature suspecte » veut s'attarder. Car cette méfiance, soulignons-le, n'est plus, de nos jours, associée uniquement à des formes de mystification qui frappent lourdement les récits de fiction - même si, de fait, les artifices trompeurs continuent d'affluer dans les textes et de prendre des tours nouveaux. De plus en plus sûrement, elle agit sur les approches de lecture critique - policière, paranoïaque, interventionniste, désobéissante -, rendant compte de la disposition dorénavant inopérante de la littérature narrative à induire l'immersion fictionnelle.

C'est qu'on « ne peut plus raconter comme avant », propose Vincent Jouve (2006), et il faut bien que cette 
impossibilité proclamée ouvre de nouvelles possibilités - c'est-à-dire relance la création : pointe les cases blanches à partir desquelles on invente la pratique, pour rappeler le dossier de la revue La lecture littéraire piloté par Marc Escola et Sophie Rabau sur la théorie littéraire et les textes possibles (2006). C'est qu'on ne peut plus lire maintenant sans voir venir les pièges. À l'éclat de la vérité, à sa quête et à son désir qui se seraient émoussés, à l'authentique et stable unité des discours, succéderaient, en fait, une constante équivoque, une fuite du sens vrai et la découverte de sens trompeurs, empilés. Ce modèle de pensée qui rend la littérature suspecte caractérise divers retours modernes lors desquels on a voulu opposer la vérité à l'illusion, la facticité à l'authentique. Ainsi, les articles que nous vous proposons examinent des œuvres de fiction à partir de ces oppositions, aident à penser l'ambiguïté, ses effets, et plus encore, la littérature comme un art qui doit ruser, déjouer, filouter, de manière à inquiéter, selon les mots de Laurent Demanze, « les frontières entre savoir stabilisé et délire » (2017).

Sur ces frontières inquiètes se développent des pratiques littéraires lucides — pour lesquelles le doute est à la fois objet d'analyse et condition de lecture -, pratiques auxquelles prennent part les articles ainsi que les « contrepoints » de notre dossier. Dans chaque proposition, on reconnaît à la fois l'herméneute qui manipule une matière friable et le créateur qui recolle les fragments de poussière.

Critiques, auteurs, lecteurs, narrateurs, personnages se penchent sur leur matériau respectif pour montrer le trou qu'on a creusé dans la vérité. Ils exposent des failles, des paradoxes; tous incapables d'embrasser ou de montrer l'étendue de leur univers, donnant à lire des fictions, des versions, si peu révélatrices... En effet, il est possible que les corpus fréquentés ici (Nicole Brossard, Alain Fleischer, Lous Hémon, Patrice Lessard, Pierre Michon, Monique Proulx, Tanguy Viel, Enrique Vila-Matas, etc.), que ces lectures offertes aussi, ne cherchent pas à représenter le monde, mais travaillent à l'opacifier.

Le constat est frappant : il vaut mieux ne pas se fier aux œuvres de fiction. Elles déroutent, confondent, multiplient les partitions, ne sont pas en mesure de donner des réponses aux questions posées par leurs silences. Sur le plan narratif, qui plus est, elles mettent à mal le contrat de « transmission » (Fortier, 2011) et instiguent des problèmes de communication (Yacobi, 1981) dont l'issue généralisée est le malentendu. Le lecteur, même, appelé à se perdre en conjectures, évite les certitudes. Averti, il étudie pourtant sa propre méfiance, s'accorde des droits sur les textes : intervient, extrapole, surinterprète. II admet faire des romans un usage impropre. Sa lecture excède les cadres, déplie les rabats, déforme les angles. II lit à côté, au verso, en dessous, par détours et raccourcis, laissant en tout temps la porte entrouverte derrière lui pour disparaître sans être vu. Quant à l'auteur, sa mauvaise foi est manifeste, et en cela, séduit (Decout, 2015). L'auteur manie l'ironie mieux que quiconque et, tapi dans l'obscurité depuis sa mort orchestrée (Barthes, 1967), profite de chaque occasion pour prendre part aux jeux d'ombres et de lumières. 
La suspicion, dans la littérature narrative qui nous occupe, se traduit de différentes manières qu'il nous intéressait de réunir selon deux perspectives pour marquer leurs implications. Ainsi éclosent d'une part, dans les corpus étudiés, des univers en trompe-l'œil, des stratégies narratives imprécises, des imaginaires exaltés, des métadiscours mensongers, des erreurs, des idées suspendues, que les contributeurs cherchent à démystifier. D'autre part, ces derniers affinent des méthodes d'investigation. Sous le couvert d'analyses, d'interprétations, de conceptualisations, ce sont presque des enquêtes auxquelles nous avons droit : ce qui défaille, déraille ou disjoncte dans les textes de fiction demande un examen scrupuleux, voire une réparation, qui compte sur des esprits analytiques tantôt tordus, tantôt obsessionnels, pour advenir. Cette perspective joueuse, du coup, donne une touche colorée aux contributions, une humeur joyeuse à l'ensemble du dossier.

En tête du numéro, Marilyn Randall interroge, dans la littérature québécoise, l'inscription de l'auteur comme figure de lui-même au sein de sa propre fiction. Ce faisant, elle réévalue la nature et le lieu de la feintise, sa destinée ludique, ainsi que la notion de partage. Le précis de Frank Wagner décline des spécimens de narrateurs antipathiques, avec pour but d'initier une réflexion sur nos dispositions à les fréquenter. Béatrice Guéna suggère une nouvelle catégorie d'auteurs : les automythomanes littéraires; les cas de Sebald, VilaMatas et Chevillard l'amènent à observer une part d'invention autofictive qui déjoue tous les contrats de lecture. Devant des romans aux motivations narratives indécidables, Cassie Bérard invite à adopter un mode de lecture conflictuelle qui consiste à épouser toutes les versions possibles. Marla Epp retrace, dans des œuvres non fiables de Michon et de Binet, les effets de fiction déconcertants, de manière à jeter le doute sur le caractère authentique des documents historiques. Louis-Daniel Godin examine un cas complexe de narration ambiguë : chez Fleischer, les voix se dédoublent pour reconstituer une histoire filiale trouée. David Bélanger et Thomas Carrier-Lafleur, enfin, déterrent, en revisitant un mythe de la littérature québécoise, le cadavre de François Paradis : leur enquête transmédiale jette la lumière sur le décès non résolu du prétendant de Maria Chapdelaine, sur lequel, étrangement, Louis Hémon fait l'impasse.

Six propositions créatives composent la section « contrepoints »: Catherine Lavarenne lève le voile sur l'entreprise de mystification d'un collectif d'auteurs responsable d'un canular à multiples facettes; Pierre Bayard, constatant le grand nombre de disparitions mystérieuses dans la littérature et le cinéma, se lance sur une nouvelle piste de recherche; Vincent Lavoie attire l'attention vers une énigmatique série de clichés documentaires déterrés par le photographe Alain Pratte; Catherine Mavrikakis se pique, dans un monologue ironique, de passer pour une auteure suspecte; Pierre-Marc Asselin, Nelly Desmarais et Marie-Pier Lafontaine concoctent à six mains un théâtre à trois voix où chaque personnage se méfie de ses comparses. La dernière proposition est le récit d'un naufrage insolite : Alec Serra-Wagneur installe une inquiétante ambiance sur un navire dont l'équipage est en proie au délire. 
Sensible aux approches de la recherche-création, l'entièreté du dossier « Littérature suspecte » vise à offrir une expérience de lecture déroutante en permettant à la fiction, comme mode de problématisation de l'imaginaire, d'infiltrer les discours critiques. Cette fiction loge dans les extrapolations, le ton et la posture avec lesquels s'engagent délibérément les contributeurs du numéro. Chacun en ses termes interroge les repères de lecture et traque les trappes au fond des placards obscurs que sont les œuvres suspectes; ces œuvres aux clés taillées pour d'autres portes, d'autres maisons, d'autres mondes que les mondes habituels.

\section{Bibliographie}

BARTHES, Roland. 1984 [1968]. « La mort de l'auteur », dans Le Bruissement de la langue. Essais critiques IV. Paris : Seuil, p. 63-69.

Decout, Maxime. 2015. En toute mauvaise foi. Sur un paradoxe littéraire Paris : Minuit, «Paradoxe », $192 \mathrm{p}$.

DemAnze, Laurent. 2017. «Petit éloge de la paranoïa. Usages déréglés de la contre-enquête». Temps zéro, 31 mars. <http://tempszero.contemporain.info/document1532>.

Escola, Marc et Sophie Rabau (dir.). 2006. Dossier « La case blanche. Théorie littéraire et textes possibles ». La lecture littéraire, no 8.

Fortier, Frances et Andrée Mercier (dir.). 2011. La transmission narrative. Modalités du pacte romanesque contemporain. Montréal : Nota Bene, «Contemporanéités », 367 p.

Jouve, Vincent. 2006. «Les métamorphoses de la lecture narrative». Protée, vol. 34, no 2-3, automne-hiver, p. $153-161$.

Sarraute, Nathalie. 1956. L'ère du soupçon. Paris : Gallimard, «Folio. Essais », 156 p.

Schaeffer, Jean-Marie. 1999. Pourquoi la fiction?. Paris : Seuil, «Poétique », 346 p.

YACOBI, Tamar. 1981. «Fictional Reliability as a Communicative Problem». Poetics Today, vol. 2, no 2 « Narratology III. Narration and Perspective in Fiction », p. 113-126.

$<$ http://www.jstor.org/stable/1772193>. 